\title{
Small cytoplasmic RNA (scRNA) gene from Clostridium perfringens can replace the gene for the Bacillus subtilis SCRNA in both growth and sporulation
}

\author{
Kouji Nakamura, Erika Hashizume, Toshinori Shibata, Yoshio Nakamura, \\ Sarka Mala and Kunio Yamane
}

Institute of Biological Sciences, University of Tsukuba, Ibaraki 305, Japan

\author{
Author for correspondence: Kouji Nakamura. Tel: +81 29853 6419. Fax: +81298536006. \\ e-mail: knakam1@sakura.cc.tsukuba.ac.jp
}

\begin{abstract}
Small cytoplasmic RNA (scRNA) is a member of an evolutionarily conserved signal-recognition-particle-like RNA family. Using a DNA fragment of Bacillus subtilis scRNA gene as a probe, we cloned and characterized a Clostridium perfringens gene encoding the SCRNA. Mapping the $5^{\prime}$ and 3 ' ends of sCRNA revealed that $C$. perfringens ScRNA consists of 269 nucleotides: the sequence has about $70 \%$ primary sequence homology with $B$. subtilis scRNA. The predicted secondary structure appeared to be similar to that of $B$. subtilis SCRNA, indicating that there are domains I and II in C. perfringens SCRNA, in addition to domain IV. Functional analysis showed that $C$. perfringens sCRNA could compensate for vegetative growth and allow the formation of heatresistant spores in an SCRNA-depleted B. subtilis strain, whereas Escherichia coli 4.5S RNA could not maintain sporulation. Since both $E$. coli 4.5S RNA and $C$. perfringens SCRNA have the same binding specificity to $B$. subtilis Ffh protein, the difference in complementation activity reflects the function of domains $I$ and II.
\end{abstract}

Keywords: Clostridium perfringens, small cytoplasmic RNA, SRP-like RNA, sporulation

\section{INTRODUCTION}

Several species of RNA, such as mRNA, rRNA and tRNA, and many small RNA forms ( $\sim 300$ nucleotides) are present in cells (Fournier \& Maxwell, 1993; Guthrie, 1991; Inouye \& Delihas, 1988). Although there is a growing list of small RNAs, their functions are little understood. Among them, signal-recognition-particle (SRP)-like RNAs have been identified in a wide range of eukaryotes, including Homo sapiens (Ullu \& Weiner, 1984), plants (Campos et al., 1988), Schizosaccharomyces pombe (Brennwald et al., 1988), and several species of Archaea (Kaine, 1990). The most studied SRP-like RNA is the mammalian 7SL RNA that is a component of the SRP (Walter \& Blobel, 1982). SRP is essential for targeting a

Abbreviations: scRNA, small cytoplasmic RNA; SRP, signal recognition particle.

The EMBL/GenBank/DDBJ accession number for the nucleotide sequences reported in this paper is D49784. subset of nascent presecretory proteins emerging from the ribosome to the endoplasmic reticulum membrane (Gilmore, 1993; Nunnari \& Walter, 1992; Rapoport, 1991, 1992; Sanders \& Schekman, 1992; Walter \& Lingappa, 1986). It has been proposed that mammalian SRP-like RNA consists of four domains (domains I-IV). In vivo, six proteins $(9,14,19,54,68$ and $72 \mathrm{kDa})$ bind to each domain (Walter \& Lingappa, 1986). Among them, the $54 \mathrm{kDa}$ GTPase protein (SRP54) binds to both the signal sequence and domain IV (High \& Dobberstein, 1991; Krieg et al., 1986; Kurzchalia et al., 1986; Romish et al., 1990; Samuelsson \& Olsson, 1993; Zopf et al., 1990). An evolutionary comparison has revealed that almost all eukaryotic and archaeal SRP-like RNAs are similar in size and can be folded into four functional domains. In contrast, bacterial SRP-like RNAs vary in size and secondary structure (Larsen \& Zwieb, 1991). Escherichia coli 4.5S RNA (114 nucleotides), which was first identified as a translational modulator (Bourgaize \& Fournier, 1987; Brown, 1987, 1989; Brown \& Fournier, 1984), folds into a single hairpin. The primary and secondary 
structures of $E$. coli $4 \cdot 5$ S RA have significant homology with that of domain IV in Homo sapiens SRP-like RNA (Poritz et al., 1988). However, this RNA lacks structures resembling domains I and III, and more than half of domain II is missing. Five other bacterial RNAs, including a 105 nucleotide RNA from the phylogenetically deepest branching Thermus thermophilus (Struck et al., 1988a), also fold into a structure similar to that of E. coli RNA. The Mycoplasma mycoides RNA (77 nucleotides) is most extensively diverged (Samuelsson \& Guindy, 1990). On the other hand, small cytoplasmic RNA (scRNA : 271 nucleotides) from Bacillus subtilis contains structures corresponding to domains I and II of the mammalian RNA (Struck \& Erdmann, 1990; Struck et al., 1988b), in addition to domain IV. We found that this structure is conserved among thirteen Bacillus species (Nakamura et al., 1992b). Functional analyses have shown that depletion of scRNA in B. subtilis led to defects in the production of extracellular enzymes and in $\beta$-lactamase translocation as well as to dramatic morphological changes (Nakamura et al., 1992a). These deficient characteristics could be compensated by introducing SRP-like RNAs from humans or E. coli, indicating that the essential function of SRP-like RNAs is evolutionarily conserved and that an essential function locates in domain IV. There is a discrepancy between the structural features of SRP-like RNAs and eubacterial phylogeny based upon 16S rRNA sequence comparisons, since $T$. thermophilus has been placed in the earliest branches of the eubacterial evolutionary tree, whereas bacilli are thought to have arisen considerably later in eubacterial evolution (Althoff et al., 1994). This lack of congruence between an established rRNA phylogeny and the size of eubacterial SRP-like RNAs begged the question as to the advantages conferred by the preservation of domains I and II upon Bacillus scRNA if eukaryotic and archaeal RNAs represent the prototype structure. To identify the structural determinants of $B$. subtilis scRNA, we constructed mutants in which individual domains or conserved nucleotides were deleted, and their importance was assayed in vivo (Nishiguchi et al., 1994). The results showed that domain IV is necessary to maintain cell viability, whereas domains I and II are not essential for vegetative growth but are needed for the formation of heat-resistant spores. Therefore, SRP-like RNA of other spore-forming eubacteria may have a similar structure to that of $B$. subtilis scRNA.

Here, we report that the secondary structure of scRNA from Clostridium perfringens appears to be similar to that of B. subtilis scRNA and that sporulation in scRNA-depleted B. subtilis can be compensated by introducing a Clostridium homologue. Therefore, it is likely that SRP-like RNA has undergone a dramatic reduction several times during bacterial evolution. However, the domains essential for sporulation remained because they conferred advantages.

\section{METHODS}

Bacterial strains and vector plasmids. Clostridium perfringens NCTC8237 was derived by $\mathrm{H}$. Hayashi, Institute of Basic Medical Sciences, University of Tsukuba, Japan. B. subtilis
SC200NA (purB6 trpB3 metB5 scr::pTUE807) was constructed as described by Nakamura et al. (1992a). In this strain, intact scRNA gene expression is regulated by the IPTG-inducible promoter Pspac-1. This strain has been maintained in the presence of $3 \mathrm{mM}$ IPTG. The expression plasmid pTUBE 809 is an E. coli-B. subtilis shuttle vector. Plasmid pTUBE809 contains a synthetic 98 bp protein A promoter of Stapbylococcus aureus. This promoter is constitutive and expressed even in the absence of IPTG. Plasmid PCR1000, into which primary products of the PCR can be cloned, was purchased from Invitrogen. The vectors PTUBE822 and pTUBE811, which can constitutively produce scRNA of $B$. subtilis and 4.5S RNA of E. coli, respectively, were constructed as described by Nakamura $e t$ al. (1992a).

Determination of the efficiency of sporulation. The efficiency of sporulation was determined as described by Nishiguchi $e t a l$. (1994). The bacterial cells were vigorously shaken in modified Schaeffer medium and harvested after $24 \mathrm{~h}$. The spores were heated at $80^{\circ} \mathrm{C}$ for $10 \mathrm{~min}$ and plated. After $12 \mathrm{~h}$, the number of cells on the plate was counted.

Construction of the plasmid vector pTUBE850. A $269 \mathrm{bp} \mathrm{DNA}$ fragment, encoding mature $C$. perfringens scRNA, was amplified from the chromosomal DNA by means of PCR with the synthetic oligonucleotides $5^{\prime}$ TGCCGTGCTAGATGGGGA $3^{\prime}$ and $5^{\prime}$ AACCGTGCACCTCACTTC $3^{\prime}$. Purified PCR product was inserted into the $H p b I$ site of the PCR1000 vector to create plasmid pTUBE840. A $315 \mathrm{bp} \mathrm{NotI-HindIII} \mathrm{fragment}$ was isolated from pTUBE840 and blunt-ended. The purified fragment was inserted between the protein A promoter and the trp transcriptional terminator. The resultant plasmid, pTUBE850, was confirmed by physical mapping and DNA sequencing.

DNA Southern hybridization. Chromosomal DNA of $C$. perfringens was prepared by the method of Saito \& Miura (1963). DNA preparations digested with restriction enzymes were separated by agarose gel electrophoresis and transferred onto a Gene Screen Plus nylon membrane (NEN Research Products). Hybridization under low stringency proceeded as described by Nakamura et al. (1990). The 271 bp DNA fragment, corresponding to mature scRNA, was amplified from the chromosomal DNA of B. subtilis 168 using PCR with the synthetic oligonucleotide primers $5^{\prime}$ TTTGCCGTGCTAAGCGGG 3' and $5^{\prime}$ TACCGTGCACCTTCTGTC $3^{\prime}$. The amplified DNA fragment was inserted at the $H p h \mathrm{I}$ site of PCR1000. After NotI-HindIII digestion, a 312 bp fragment was isolated. This fragment was labelled with $\left[\alpha^{-32} \mathrm{P}\right] \mathrm{dCTP}$ using a random primer labelling kit (Takara Shuzo) and used as a probe.

DNA sequencing analysis. The nucleotide sequence of the entire $2.7 \mathrm{~kb}$ HindIII fragment containing the scRNA gene was determined. The fragment was first degraded by sonic oscillation, then $0.5-1 \mathrm{~kb}$ fragments were isolated. After repairing both ends with Klenow large fragment and T4 DNA polymerase, the fragments were inserted into the SmaI site of pUC119. DNA fragments in the recombinant plasmids were amplified and sequenced by dideoxy-chain termination using an automatic DNA sequencer type 373A (Perkin-Elmer Japan). Both strands were sequenced at least three times, and all ends of the fragments overlapped. The primary sequences were edited and analysed by GENETYX-MAC (Softwate Development Company, Tokyo, Japan). The DNA sequences were compared by sending them to the blast-mail server at the Human Genome Center at Tokyo University.

Mapping of both ends of scRNA transcripts. Total RNA of $C$. perfringens was extracted as described by Nakamura et al. (1992a). 
Primer P-1 (5' CACAAGTTAGAGCCTCTATG 3'), positions 352-371 (Fig. 2), was used to precisely map the $5^{\prime}$ end of mature scRNA. Total $C$. perfringens RNA $(40 \mu \mathrm{g})$ and $5 \times 10^{4}$ c.p.m. of the ${ }^{32} \mathrm{P}$-labelled oligonucleotide primer were hybridized at $40^{\circ} \mathrm{C}$ overnight. After hybridization, the sample was precipitated by adding two vols ethanol and being stored at $20^{\circ} \mathrm{C}$ for $1 \mathrm{~h}$. The pellet was collected by centrifugation and dissolved in $20 \mu \mathrm{l}$ reverse transcriptase buffer $(50 \mathrm{mM}$ Tris/ $\mathrm{HCl}, \mathrm{pH} 7 \cdot 6,60 \mathrm{mM}$ $\mathrm{KCl}, 10 \mathrm{mM} \mathrm{MgCl}, 1 \mathrm{mM}$ each dNTPs, $1 \mathrm{mM}$ DT'T). Fifty units of RAV-2 (Rous associated virus 2) reverse transcriptase was added and the mixture was incubated at $42{ }^{\circ} \mathrm{C}$ for $1 \mathrm{~h}$. The length of extended DNA fragment was determined by a comparison with sequencing ladders generated from M13mp10 containing the $C$. perfringens scRNA gene using the same oligonucleotides. The $3^{\prime}$ end of scRNA was estimated by the RNase protection assay. A $332 \mathrm{bp} A p a I-E c o R I$ fragment, including the $3^{\prime}$ portion of the $C$. perfringens scRNA (Fig. 1), was inserted between the $A p a \mathrm{I}-\mathrm{E} c 0 \mathrm{RI}$ sites of Bluescript SK $(-)$. T3 RNA polymerase and $\left[\alpha-{ }^{32} \mathrm{P}\right] C T P$ were used to synthesize a radioactive probe which encompassed the $3^{\prime}$ portion of the scRNA gene and to which it is complementary. The RNase protection assay was performed as described by Nakamura et al. (1992a) using total RNA $(10 \mu \mathrm{g})$ and $3.9 \times 10^{6}$ c.p.m. of the ${ }^{32}$ P-labelled probe.

Synthesis of ${ }^{32}$ P-labelled SRP-like RNAs from B. subtilis, E. coli and $C$. perfringens. The DNA fragments including domain IV of SRP-like RNAs from three bacteria were placed under the control of the SP6 promoter. A $114 \mathrm{bp}$ AluI-HincII fragment, corresponding to positions +109 to +222 of $B$. subtilis scRNA, was isolated and inserted into the Smal site of pSP64 in the correct direction. A 138 bp DNA fragment which encodes mature E. coli $4.5 \mathrm{~S}$ RNA was amplified with the synthetic oligonucleotides $5^{\prime}$ AAGCTTAAGCT'TGGGGGCTCTGTTGGTTCT 3 ' and 5' GGATCCGGATCCGGGTGGGGGCCCTGCCAGCT 3'. A 141 bp DNA fragment, encoding from positions +100 to +216 of $C$. perfringens scRNA, was amplified with the oligonucleotides $5^{\prime}$ AAGCTTAAGCTTATGTAAGTGGTGT'TGAGA 3' and 5' GGATCCGGATCCTCAAGCCAGACGTATCCA $3^{\prime}$. These four primers were designed to create HindIII and BamHI sites at the $5^{\prime}$ and $3^{\prime}$ ends of the resulting PCR products, respectively. Purified products were first digested with both HindIII and $B a m H I$, then inserted between the HindIII and BamHI sites in PSP64. To prepare a radioactive probe used for the RNA mobility shift assay, the resulting plasmids were linearized by digestion with $B a m \mathrm{HI}$, and used as a transcriptional template. The transcription reaction contained $40 \mathrm{mM}$ Tris $/ \mathrm{HCl}(\mathrm{pH} 8), 6 \mathrm{mM} \mathrm{MgCl}$, $5 \mathrm{mM}$ DTT, $1 \mathrm{mM}$ spermidine, $0.01 \%$ Triton X-100, $50 \mu \mathrm{g}$ BSA ml ${ }^{-1}, 2 \mathrm{mM}$ each of ATP, GTP and UTP, $0.2 \mathrm{mM}$ CTP, 20 units RNasin, $50 \mu \mathrm{Ci}(1.85 \mathrm{MBq})\left[\alpha_{-}{ }^{32} \mathrm{P}\right] \mathrm{CTP}\left(3000 \mathrm{Ci} \mathrm{mmol}^{-1}\right.$; $111 \mathrm{TBq} \mathrm{mmol}^{-1}$ ), 25 pmol DNA fragment, and 200 units SP6 RNA polymerase (Takara Shuzo). The specific activity of the RNA product was $\sim 3 \times 10^{7}$ c.p.m. $\mu \mathrm{g}^{-1}$. It was purified as a single band after PAGE.

RNA mobility shift assay. ${ }^{32}$ P-labelled RNAs $\left(10^{4}\right.$ c.p.m.), corresponding to domain IV of each bacterial SRP-like RNA, were incubated with various amounts of purified $B$. subtilis $\mathrm{Ffh}$ protein $(0,0.2,0 \cdot 4,0.6$ and $0.8 \mu \mathrm{g}$, as indicated in the legend to Fig. 7) in $20 \mu \mathrm{l}$ reactions containing $10 \mathrm{mM}$ Tris $/ \mathrm{HCl}(\mathrm{pH} \mathrm{7.5)}$, $80 \mathrm{mM} \mathrm{KCl}, 5 \mathrm{mM} \mathrm{MgCl}, 0 \cdot 1 \%$ Triton X-100, $1 \mathrm{mM}$ DTT, $1 \mathrm{mM}$ EDTA, 4 units RNasin, $5 \%$ (v/v) glycerol and $100 \mathrm{ng}$ poly(dI-dC). After a $40 \mathrm{~min}$ incubation at room temperature, samples were loaded onto a $8 \%(\mathrm{w} / \mathrm{v})$ nondenaturing polyacrylamide gel containing $45 \mathrm{mM}$ Tris $/ \mathrm{HCl}(\mathrm{pH} 8.3)$ and $12.5 \mathrm{mM}$ EDTA. The gel was dried, then visualized by autoradiography.

\section{RESULTS \\ Cloning of the scRNA gene of the $C$. perfringens chromosome}

We identified the scRNA gene from $C$. perfringens by means of Southern hybridization using a 312 nucleotide DNA fragment encoding the mature $B$. subtilis scRNA

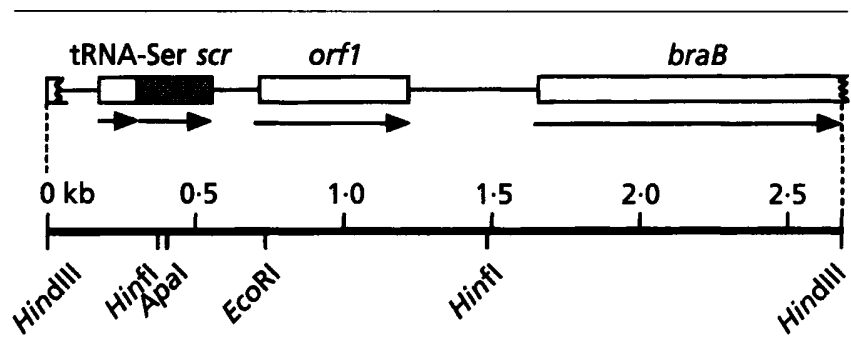

Fig. 1. Physical map and gene organization in the cloned $2.7 \mathrm{~kb}$ HindIII fragment from $C$. perfringens chromosomal DNA. The locations of the four genes (tRNA-Ser, scr, orf1 and braB) are indicated by the rectangles above the physical map. The shadowed rectangle represents the scr gene. Arrows indicate the direction of transcription in each gene.

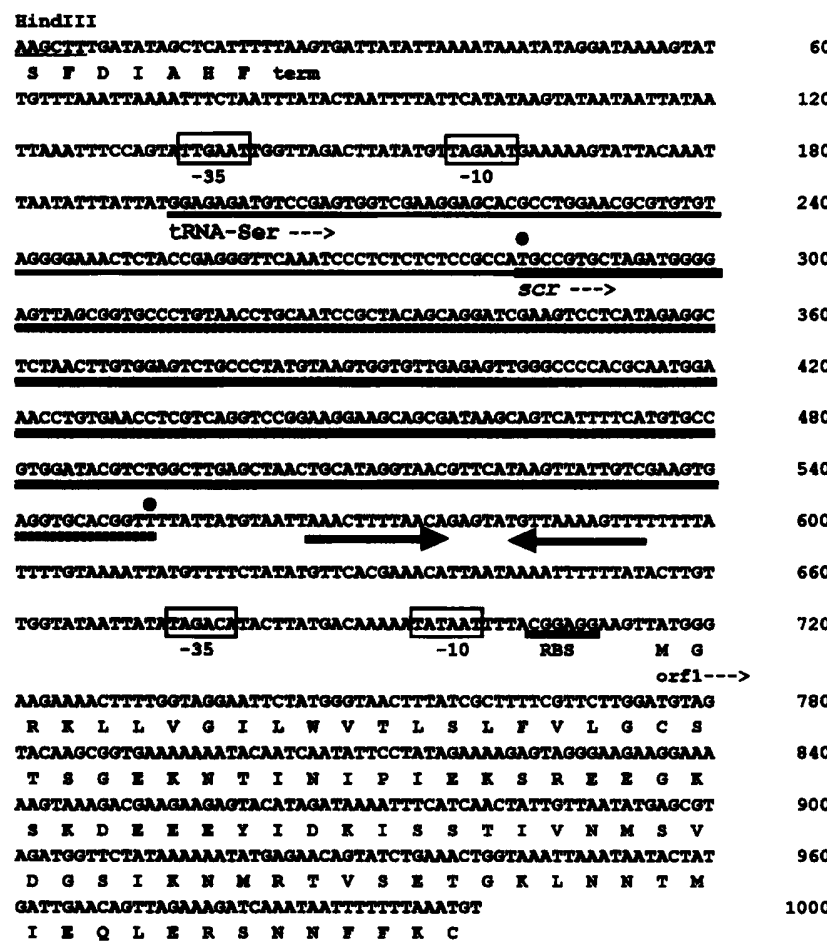

Fig. 2. A $1 \mathrm{~kb}$ nucleotide sequence of the $2.7 \mathrm{~kb}$ HindIII fragment cloned. The nucleotides are numbered from the $5^{\prime}$ end, the first being taken as a Hindlll restriction site. The presumed promoter elements $(-10$ and -35$)$ are boxed. The potential ribosome-binding sites (RBS) are underlined. Palindromic sequences that may serve as transcriptional terminators are indicated by converging arrows. The $5^{\prime}$ and $3^{\prime}$ ends of sCRNA, shown in Fig. 4, are marked by a dot above the corresponding nucleotides $(+284$ and +552$)$. The sequences exhibiting homology with tRNA-Ser and SCRNA of $B$. subtilis are thickly underlined. 
(a)

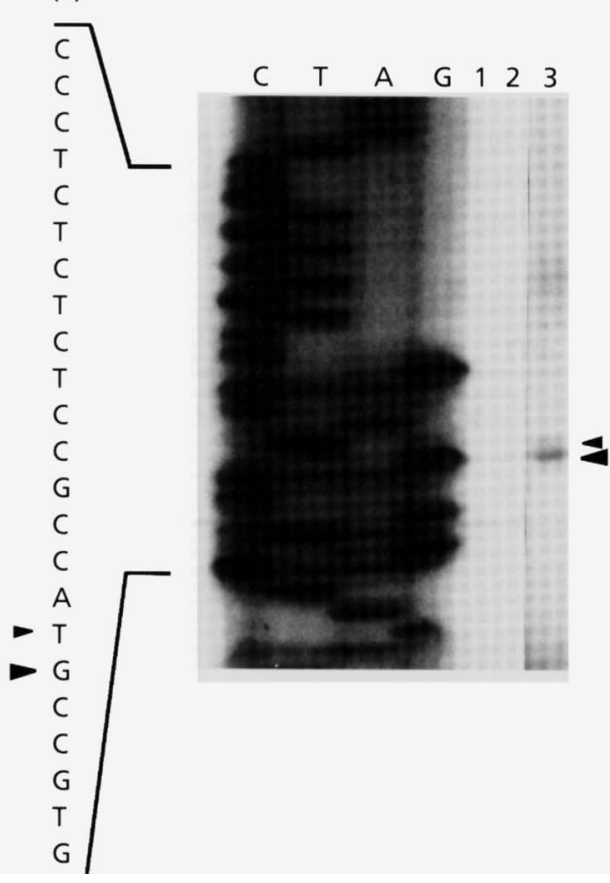

(b)

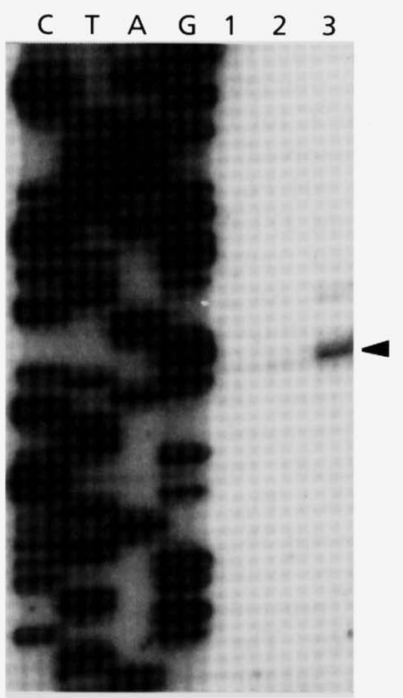

Fig. 3. Determination of the $5^{\prime}$ and $3^{\prime}$ ends of $C$. perfringens scRNA. (a) Mapping the $5^{\prime}$ terminus by primer extension. Lanes $C, T, A$, and $G$ show DNA sequence ladders primed by the oligonucleotide used for primer extension of the RNA from $C$. perfringens. Total RNA samples were prepared from $C$. perfringens cultured for 1 (lane 1), 2 (lane 2), and 3 (lane 3 ) $h$ after inoculation. The large arrowhead marks the primer extension product with the 5'-labelled oligonucleotide. The small arrowhead corresponds to a minor band. (b) Mapping the $3^{\prime}$ terminus by means of an RNase protection assay of the scRNA gene transcript. RNA samples are the same as those used in (a). The arrowhead indicates the protected product. (c) Proposed secondary structure of the $3^{\prime}$ portion of the SCRNA gene, highlighting the secondary structure that may serve as a $\rho$-independent terminator. The arrowhead indicates the $3^{\prime}$ endpoint, as proposed in (b).

gene as a probe. A $2.7 \mathrm{~kb}$ HindIII fragment specifically hybridized with the probe (data not shown). DNA fragments of 2-4 kb were extracted from the gel and inserted into the HindIII site of pUC119. Among 400 plasmids that we purified, we found two clones showing positive bands. Restriction mapping the plasmids in the two positive clones revealed that both contained a fragment of about $2 \cdot 8 \mathrm{~kb}$, oriented in the same direction as the vector. One of them was used for determining the sequence.

\section{Sequencing and characterization of the chromosome region around the scRNA gene of $C$. perfringens}

We sequenced the entire $2 \cdot 7 \mathrm{~kb}$ fragment to which the $B$. subtilis scRNA gene was hybridized. The physical map of the $2.7 \mathrm{~kb}$ fragment is shown in Fig. 1. The nucleotide sequence and deduced amino acid sequence of a $1000 \mathrm{bp}$ fragment around the scRNA gene is shown in Fig. 2. Two ORFs were found in this fragment. They were aligned in the same direction and no sizeable ORF was found in the opposite direction. The first ORF, starting with an ATG codon at positions 716-718, consisted of 167 codons and would encode a protein of $18.4 \mathrm{kDa}$. This protein exhibited no significant homology to known proteins in the protein-sequence library at the Human Genome Center at Tokyo University. The second ORF begins with an ATG codon at positions 1659-1661. However, no termination codon was found in the downstream region. Therefore, we could not isolate the entire ORF. The amino acid sequence of this truncated protein showed $34.8 \%$ amino acid identity with the $\mathrm{N}$-terminal portion of the product of the sodium-coupled, branched-chain amino acid carrier gene $(b r a B)$ of Pseudomonas aeruginosa (Hoshino et al., 1990). Therefore, this gene was designated as $b r a B$ in $C$. perfringens. There are typical sequences for the ribosome-binding sites in front of these ORFs.

From a database search of the DNA sequences, the nucleotide sequence from positions 284 to 552 showed $73.2 \%$ identity to that of the B. subtilis scRNA gene. We also found that a secondary structure of a gene juxtaposed to the scRNA gene had a typical clover-leaf structure conserved in the tRNA structure and that all determinant nucleotides conserved in tRNA-Ser (Normanly et al., 1986), including the discriminator base, are well preserved in this tRNA gene. The sequence of the anti-codon of this 

C. perfringens
B. subtilis
T. thermophilus
E. coli
P. aeruginosa

--TGCCGTGCTAGATGGGGAGTTAGCGGTGCCCTGTAACCTGCAATCCGCTACAGCAGGA TTTGCCGTGCTAAGCGGGGAGGTAGCGGTGCCCTGT-ACCTGCAATCCGCTCTAGCAGGG
C. perfringens
B. subtilis
T. thermophilus
E. coli
P. aeruginosa

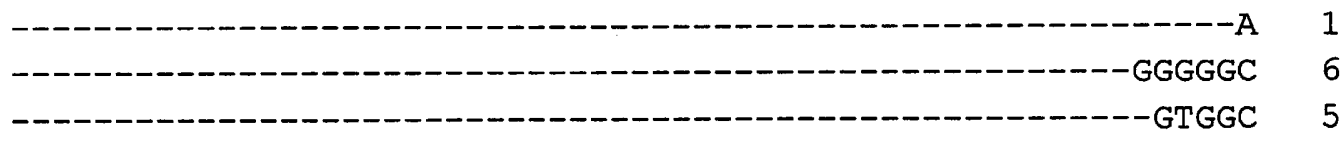

\section{C. perfringens \\ B. subtilis \\ T. thermophilus \\ E. coli \\ P. aeruginosa}

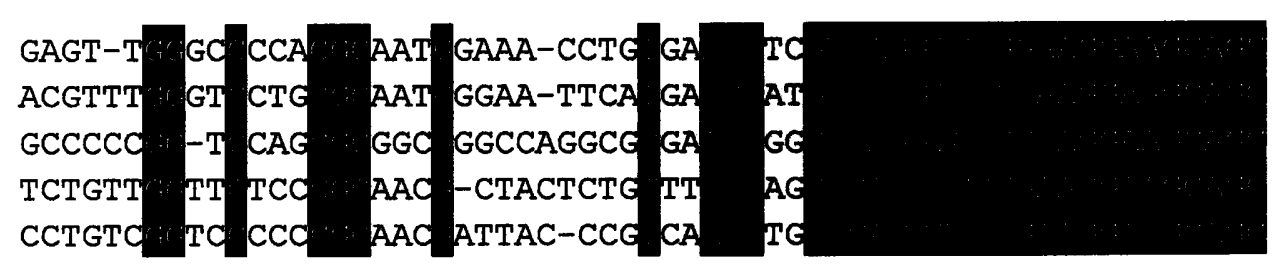

\section{C. perfringens \\ B. subtilis \\ T. thermophilus \\ E. coli \\ P. aeruginosa}

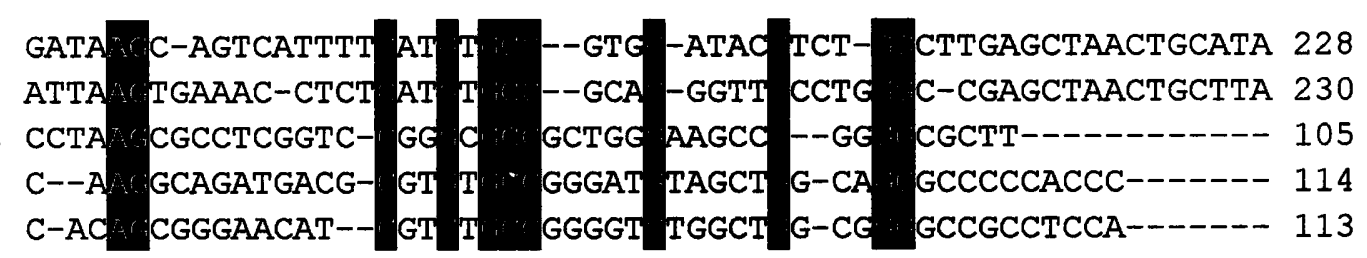
C. perfringens
B. subtilis
T. thermophilus
E. coli
P. aeruginosa

GGTAACGTTCATAAGTTATTGTCGAAGTGAGGTGCACGGTT

Fig. 4. Alignment of $C$. perfringens scRNA with other eubacterial cognates. The sequence of $C$. perfringens scRNA was aligned with SRP-like RNAs from B. subtilis (accession no. X06802), T. thermophilus (X12643), E. coli (X01074) and P. aeruginosa (M31829). The identical residues among all sequences are indicated by reversed-out letters. Dashes indicate gaps for maximal homology. The nucleotide sequence was aligned with other sequences for maximal homology, using the multiple alignment feature of the GENETYX-MAC software.

tRNA is GGA, demonstrating that this gene encodes tRNA-Ser. About 30 bp upstream from the tRNA-Ser gene a potential promoter was identified ( -35 box, TTGAAT; -10 box, TAGAAT), which was highly homologous with the consensus sequence recognized by the E. coli and B. subtilis $\sigma^{\mathrm{A}}$ RNA polymerase ( -35 box, TTGACA; -10 box, TATAAT) (Gitt et al., 1985). About 12 bp downstream from the scRNA coding region there is an almost perfect palindrome sequence comprising 29 bp $\left[\Delta G=-13.4 \mathrm{kcal} \mathrm{mol}^{-1}\left(-56.28 \mathrm{~kJ} \mathrm{~mol}^{-1}\right)\right]$ as calculated by the method of Tinoco et al. (1973) followed by a run of thymidine residues (Fig. $3 c$ ). This structure resembled that of a characteristic $E$. coli $\rho$-independent transcriptional terminator. Furthermore, between the tRNA-Ser and scRNA genes there was no gap, indicating that these two genes are organized into an operon and that the two genes are transcribed as one primary transcript.

\section{Mapping of $5^{\prime}$ and $3^{\prime}$ ends for SCRNA of $C$. perfringens}

To verify the structure of scRNA from $C$. perfringens, both the $5^{\prime}$ and $3^{\prime}$ ends were determined. In addition, we investigated whether or not processing events occurred in $C$. perfringens scRNA, since the processing at the $5^{\prime}$ and $3^{\prime}$ ends is involved in the maturation of the $B$. subtilis scRNA. To determine the $5^{\prime}$ end of scRNA, we isolated RNA from $C$. perfringens cells cultured in GAM broth (Nissui) under anaerobic conditions 1, 2 and $3 \mathrm{~h}$ after inoculation. The P-1 primer detected a major band with 

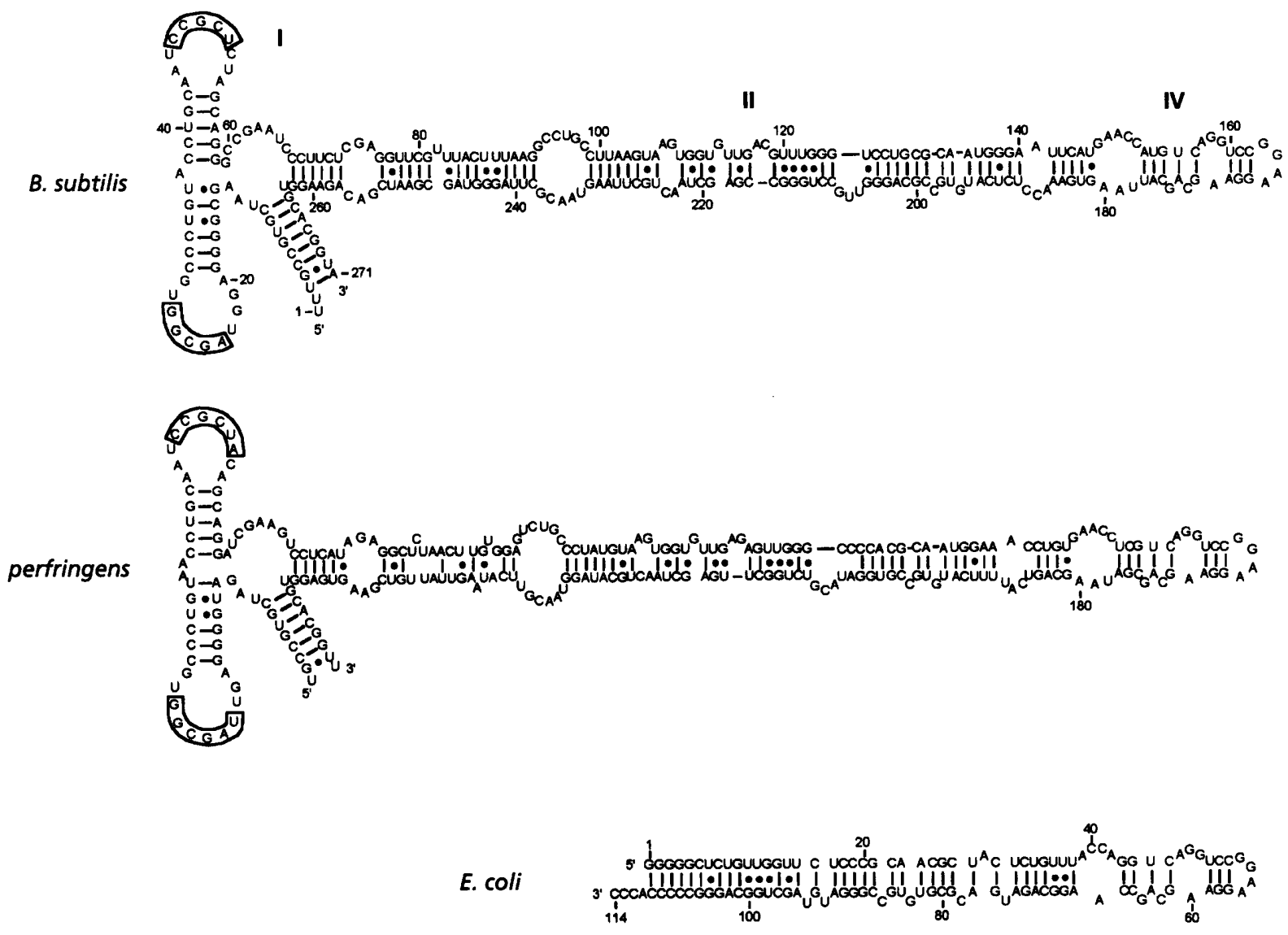

Fig. 5. Proposed secondary structures of $B$. subtilis scRNA (top), C. perfringens scRNA (centre) and E. coli 4.55 RNA (bottom). GU base pairs are indicated by open circles. Domains I, II and IV of $B$. subtilis are indicated. Nucleotides that are involved in the potential interaction between two loops within domain I are boxed.

the $5^{\prime}$ end located at the $\mathrm{G}$ residue at position 285 (Fig. $3 a$ ). Moreover, a minor band (about $10 \%$ of the intensity of the major signal) that has a $5^{\prime}$ end located 1 nucleotide upstream of the major band was also detected. These data indicated that the $5^{\prime}$ end of scRNA starts from the T residue at nucleotide position 284. This lack of 1 nucleotide at the $5^{\prime}$ end is probably an artifact caused during RNA preparation or during the primer extension reaction. For the $3^{\prime}$-end mapping, a 332 bp $A p a \mathrm{I}-E c o \mathrm{RI}$ fragment (Fig. 1) was inserted into Bluescript $\mathrm{SK}(-)$. The radioactive antisense RNA was prepared as described in Methods. Fig. 3(b) shows that only one major protected band, comprising $146 \mathrm{bp}$, appeared in the RNA sample prepared at $3 \mathrm{~h}$ after inoculation (Fig. 3b, lane 3). This result indicates that the $3^{\prime}$ end of scRNA is a T residue at nucleotide position 552 in Fig. 2. A $5^{\prime}$ and $3^{\prime}$ mapping study showed that the mature scRNA of $C$. perfringens scRNA consists of 269 nucleotides. In contrast to the SRP-like RNAs from E. coli, $T$. thermopbilus and $P$. aeruginosa, the scRNA of $C$. perfringens can be significantly aligned with the entire region of B. subtilis scRNA (Fig. 4), with which it has about $70 \%$ homology. Moreover, based upon the secondary structure predicted in $B$. subtilis scRNA, most of the nucleotide substitutions observed in C. perfringens scRNA are covariant. Therefore, a similar secondary structure can be shown for $C$. perfringens scRNA (Fig. 5). In addition to the evolutionarily conserved domain IV, scRNA of $C$. perfringens has both domains I and II. This is a second example of a bacterial SRP-like RNA that contains these domains.

\section{C. perfringens sCRNA can compensate for the vegetative growth and sporulation of SCRNA- depleted B. subtilis cells}

Because of the structural resemblance, we examined whether or not $C$. perfringens scRNA functionally relates to B. subtilis scRNA. The test strain, a B. subtilis SC200NA, contains two scRNA gene (scr) loci. One allele is the inducible Pspac-1::scr gene introduced by fusing the chromosomal scr locus with the spac-1 promoter by homologous recombination. The other is an inactive variant of the normal scr gene that lacks the $3^{\prime}$ one-third of the gene. Repression of the Pspac-1 allele is strengthened in the absence of IPTG. Without IPTG, this cell is not viable and has aberrant morphological features during the early period of incubation. We introduced $B$. subtilis $-E$. coli shuttle plasmids, containing the gene for RNA homologues from $B$. subtilis (pTUBE822), E. coli (pTUBE811) and $C$. perfringens (pTUBE850), into $B$. 


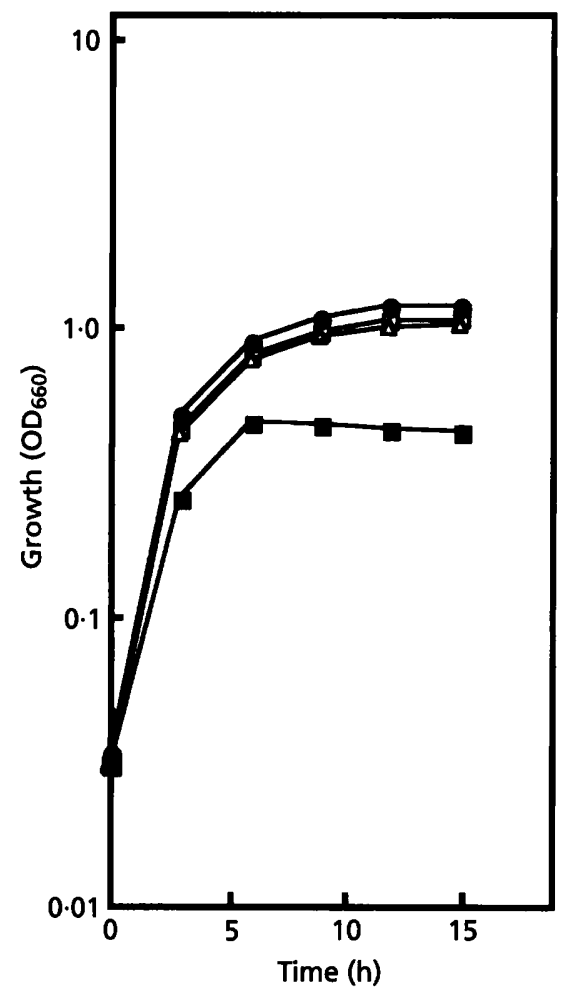

Fig. 6. Complementation of cell growth of B. subtilis strains in the depletion of intrinsic scRNA by introduction of eubacterial SRP-like RNA homologues. B. subtilis SC200NA strains harbouring vector pTUBE809 ( $\square$ ), pTUBE822 (O), pTUBE811 ( $\square$ ) or pTUBE850 $(\triangle)$ were cultured in L-broth at $37^{\circ} \mathrm{C}$ in the absence of IPTG. The data shown are the means of two experiments performed on different days.

subtilis SC200NA. As shown in Fig. 6, the growth rates at the exponential and early stationary phases are indistinguishable among the three transformants, indicating that these RNAs are functionally interchangeable. This result is consistent with those of previous studies demonstrating compatibility within the SRP-like RNA family (Brown et al., 1989; Brown, 1991; Nakamura et al., 1992a; Simoneau \& Hu, 1992). However, we also showed that a B. subtilis SC200NA transformant containing mutant
scRNA, in which domain I and/or II is depleted, can no longer sporulate with the same frequency as the wild-type (Nishiguchi et al., 1994). Therefore, it is of interest to determine whether $C$. perfringens and E. coli RNAs can compensate for formation of heat-resistant spores of SC200NA in the absence of IPTG. To determine the sporulation efficiency, each transformant was cultivated in Schaeffer sporulation medium for $24 \mathrm{~h}$ without IPTG. As indicated in Table 1, sporulation of the pTUBE822 transformant was heavy, while that of the pTUBE809 transformant was suppressed (Table 1). Under these conditions, the pTUBE850 transformant sporulated and had nearly the same frequency of heat-resistant spores as the pTUBE822 transformant. On the other hand, the frequency of that of the pTUBE811 transformant, which expresses E. coli $4.5 S$ RNA, was reduced to about $40 \%$ of that of the pTUBE822 transformant. This frequency is similar to that of the pTUBE823 transformant, which expresses an scRNA variant lacking domain I (Nishiguchi et al., 1994). Quantification of the RNAs expressed in the $B$. subtilis transformants confirmed that E. coli $4.5 \mathrm{~S}$ RNA accumulated as well as $B$. subtilis and $C$. perfringens scRNAs (data not shown), indicating that this reduced frequency in the pTUBE811 transformant is not due to instability of the RNA.

\section{Ability of SRP-like RNAs from three bacteria to bind to $B$. subtilis Ffh protein}

We elucidated that scRNA binds to the GTPase protein Ffh in vivo to form a stable ribonucleoprotein complex (Nakamura et al., 1994). Ffh protein is a homologue of mammalian SRP54 (Bernstein et al., 1989; Hann et al., 1989; Honda et al., 1993; Samuelsson, 1992). In contrast to mammalian SRP54, bacterial homologues have an intrinsic GTPase activity (Samuelsson \& Olsson, 1993). Since complex formation between Ffh and scRNA is considered to be important for scRNA to function properly, it is possible that the depleted ability of E. coli 4.5S RNA to bind to Ffh led to a reduction in the efficiency of sporulation. To test this hypothesis, the ability of each RNA to bind to Ffh protein was determined by the RNA mobility shift assay. Since full size $B$. subtilis and $C$. perfringens scRNAs are considered too long for use

Table 1. Efficiency of heat-resistant spore formation in SC200NA containing each scRNA gene

The data shown are the means of three experiments performed on different days.

\begin{tabular}{|c|c|c|c|c|}
\hline Plasmid & $\begin{array}{l}\text { Origin of } \\
\text { scRNA }\end{array}$ & $\begin{array}{c}\text { Viable cells } \\
\left(10^{-8} \times \text { c.f.u. } \mathrm{ml}^{-1}\right)\end{array}$ & $\begin{array}{c}\text { Heat-resistant } \\
\text { spores } \\
\left(10^{-8} \times \text { c.f.u. } \mathrm{ml}^{-1}\right)\end{array}$ & $\begin{array}{l}\text { Frequency } \\
\qquad(\%)^{*}\end{array}$ \\
\hline pTUBE809 & None & $2 \cdot 5$ & $0 \cdot 1$ & 4 \\
\hline pTUBE822 & B. subtilis & $4 \cdot 2$ & $2 \cdot 5$ & 60 \\
\hline pTUBE850 & C. perfringens & 3.9 & $2 \cdot 3$ & 59 \\
\hline pTUBE811 & E. coli & $3 \cdot 2$ & 0.8 & 25 \\
\hline
\end{tabular}

*Value for heat-resistant spores/value for viable cells $\times 100$. 

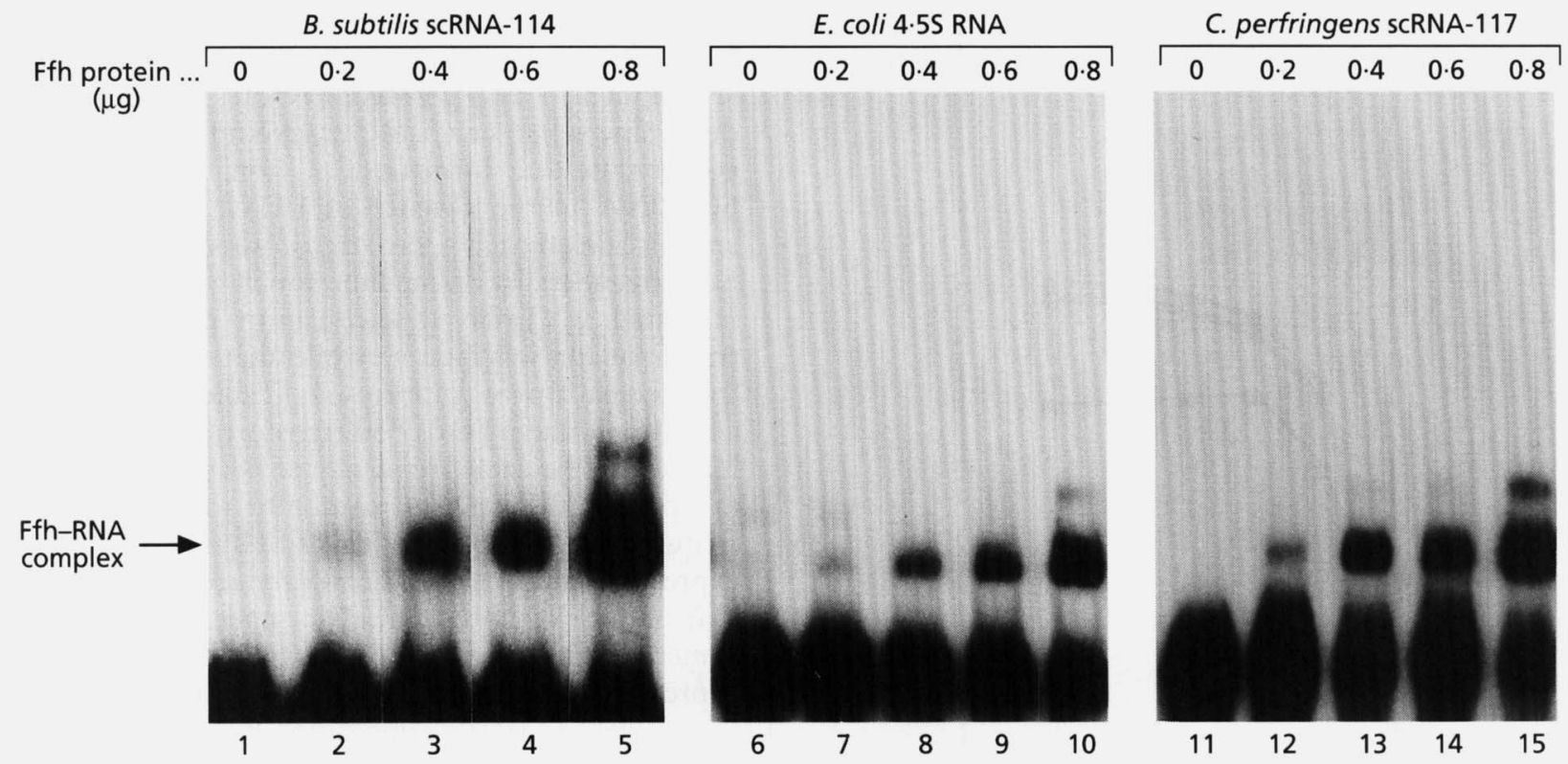

Fig. 7. Binding activity of $B$. subtilis scRNA-114 (left), E. coli 4.5S RNA (centre) and C. perfringens scRNA-117 (right) with $B$. subtilis Ffh protein. Each ${ }^{32}$ P-labelled RNA was incubated with no added protein (lanes 1, 6 and 11), or with 0.2 (lanes 2, 7 and 12), 0.4 (lanes 3, 8 and 13), 0.6 (lanes 4,9 and 14) or 0.8 (lanes 5,10 and 15) $\mu$ g protein as described under Methods. The arrow indicates the RNA-protein complex.

as a probe for this assay and truncated $B$. subtilis scRNA corresponding to domain IV can efficiently bind to Ffh protein (data not shown), we used the radioactive truncated form of each RNA, namely B. subtilis scRNA114 and $C$. perfringens scRNA-117. These RNAs correspond to positions +109 to +222 of $B$. subtilis scRNA (B. subtilis scRNA-114) and positions +100 to +216 of $C$. perfringens scRNA (C. perfringens scRNA-117), respectively. The RNA secondary structure predicted using the Zucker minimal free energy program (Zucker, 1989) revealed that these RNAs can form a single hairpin structure just like $E$. coli $4.5 \mathrm{~S}$ RNA. Furthermore, the expression of $B$. subtilis scRNA-114 and $C$. perfringens scRNA-117 complemented the growth of $B$. subtilis SC200NA in the absence of IPTG (data not shown). Fig. 7 demonstrates that $B$. subtilis scRNA-114 ${ }^{32} \mathrm{P}$-labelled RNA that mimics domain IV bound to Ffh protein in the mobility shift assay (lanes 1-5). Unlabelled B. subtilis scRNA-114, but not tRNA, diminished this complex formation (data not shown), exhibiting binding specificity in this assay. Both E. coli $4.5 \mathrm{~S}$ RNA and $C$. perfringens scRNA-117 also associate with Ffh protein and brought about the appearance of a complex form (lanes 6-10 and lanes 11-15). The amount of complex formed was dependent on the amount of protein added. The binding specificity of this complex was demonstrated with various unlabelled RNAs (data not shown). Quantitative densitometry of autoradiograms showed that the intensity of the complex that appeared in E. coli $4.5 \mathrm{~S} \mathrm{RNA}$ and $C$. perfringens scRNA-117 was about $95 \%$ of that in $B$. subtilis scRNA-114. These results demonstrated that $E$. coli $4 \cdot 5 \mathrm{~S}$ RNA and $C$. perfringens scRNA-117 interacted with $B$. subtilis $\mathrm{Ffh}$ protein in vitro and exhibited the same apparent specificity for B. subtilis scRNA-114.

\section{DISCUSSION}

The genus Clostridium consists of a diverse collection of obligatory anaerobic Gram-positive bacteria that all sporulate (Smith \& Hobbs, 1974). Based upon a phylogenetic study derived from a comparison of the $16 \mathrm{~S}$ and 23S rRNA sequences, Clostridium is related to the enlarged Bacillus cluster (van der Meer et al., 1993; Woese, 1987). Clostridium spp. have a dG $+\mathrm{dC}$ content varying from 24 to $55 \%$. C. perfringens has been studied intensively because of its importance in human pathogenesis (Finegold, 1977) and it has a high $\mathrm{dA}+\mathrm{dT}$ content $(\sim 75 \%)$. Here, we reported that $C$. perfringens has a DNA sequence that is significantly homologous with the entire region of $B$. subtilis scRNA. A functional analysis showed that this encodes an scRNA in $C$. perfringens. The scRNAs of $C$. perfringens and B. subtilis were almost the same size (269 and 271 nucleotides) and shared a comparatively high degree of identical nucleotides $(73 \cdot 2 \%)$. This relationship led us to construct a common secondary structural model for the two scRNAs. This model indicated that $C$. perfringens scRNA has both domains I and II, in addition to domain IV. The secondary structure is supported by several compensatory base-changes identified by a phylogenetic comparison. Moreover, as shown in Fig. 5, there is a potential tertiary interaction between two loops located within domain I of B. subtilis. This interaction can be maintained in $C$. perfringens scRNA and 6 nucleotides are involved in this interaction.

As shown in Results, the tRNA-Ser and scRNA genes are organized into an operon. Haas et al. (1990) have reported that the 7S RNA gene locates upstream of the tRNA-Ser gene and they detected transcripts of the 7S RNA and tRNA-Ser genes in the extremely thermophilic archaeo- 
bacterium Methanothermus fervidus. On the other hand, in trypanosomes, upstream tRNA genes are essential for $7 \mathrm{~S}$ RNA gene expression (Nakaar et al., 1994). However, since the scRNA gene of $B$. subtilis is a monofunctional transcriptional unit (Struck et al., 1989), we cannot underscore the significance of the association of tRNA and SRP-like RNA genes. The endoribonuclease P is responsible for generating the $5^{\prime}$ termini of tRNAs (Altman, 1989). On the other hand, knowledge of the $3^{\prime}$ processing of the tRNA precursor is severely limited (Deutscher, 1990). Early in vitro studies of the processing of a few E. coli tRNA precursors led to suggestions that the generation of a mature $3^{\prime}$ terminus requires several multiple exoribonuclease activities. Several studies with mutant tRNA precursors or with extracts deficient in exoribonucleases supported the notion that the generation of the mature $3^{\prime}$ terminus of tRNA involves an initial endonucleolytic cleavage downstream of the encoded -CCA sequence, followed by exonucleolytic trimming to expose these residues (Bikoff \& Gefter, 1975; Sekiya et al., 1979). In contrast, there is no spacer region between the tRNA-Ser and scRNA genes of $C$. perfringens. Our data indicated that only a one-step endonucleolytic cleavage after the A residue at +283 (Fig. 3) is required to create the mature $3^{\prime}$ end of tRNA. Therefore, although the ribonucleases involved in the processing are unknown, this structural feature represents the unique manner of the 3 -end processing of the tRNA transcript.

In contrast to the unification in the size and secondary structure of SRP RNA in eukaryotes, except for the yeast homologue, that of bacterial SRP-like RNAs has diverged (Larsen \& Zwieb, 1991), i.e. E. coli $4.5 S$ RNA is 114 nucleotides, $P$. aeruginosa is $113, T$. thermophilus is $105, B$. subtilis is $271, C$. perfringens is 269 and M. mycoides is 77 . Among them, both $B$. subtilis and $C$. perfringens contain structures corresponding to domains I, II and IV of mammalian 7SL RNA. Since both organisms are Grampositive and can sporulate, the simplest explanation for these data is that the persistence of these structures reflects the close relationship between $B$. subtilis and C. perfringens. However, our data show that $S$. aureus, being more closely related to Bacillus species based on both $16 \mathrm{~S}$ and $23 \mathrm{~S}$ rRNA phylogeny, has SRP-like RNAs, the size of which are intermediate between $B$. subtilis and $E$. coli (E. Hashizume and others, unpublished). Moreover, T. Barry and others (personal communication) have determined the sequence of Listeria monocytogenes SRP-like RNA. $L$. monocytogenes is a Gram-positive bacterium that does not sporulate. The size and secondary structure of Listeria spp. RNA resembles that of $B$. subtilis scRNA, but there are several base substitutions that disrupt the secondary structure of domains I and II. Furthermore, the potential interaction between two loops within domain $I$ has vanished. As a result, the SRP-like RNA of $L$. monocytogenes contains three domains but the secondary structure of domain $I$ is ambiguous. To examine the functions of domains I and II, we constructed mutant scRNAs of $B$. subtilis in which individual domains or conserved nucleotides were deleted, and examined their effects in vivo. The results demonstrated that domains I and II have distinct functions in the formation of heat-resistant spores (Nishiguchi et al., 1994). Here, we showed that introducing $C$. perfringens scRNA in the conditional scRNA-deficient mutant B. subtilis SC200NA can compensate for not only the vegetative growth but also for heat-resistant spore formation, whereas E. coli $4.5 \mathrm{~S}$ RNA was significantly less effective in supporting sporulation. This difference in activity is not due to the different ability of each RNA to bind to B. subtilis $\mathrm{Ffh}$ protein. The conservation of domains I and II throughout eubacterial evolution is exclusively related to the requirement for their functions in both organisms. We thus suggest a plausible scenario for the evolution of SRP-like RNA in cells of bacterial lineage. If eukaryotic and archaeobacterial SRP-like RNAs represent features of their prototype (Althoff $e t$ al., 1994), then SRP-like RNA has undergone several dramatic size reductions during bacterial evolution. Moreover, this phenomenon could occur at each step of evolution, even after the species $B$. subtilis. E. coli and Clostridium appeared. However, the domains needed for efficient sporulation conferred selective advantages in $B$. subtilis and $C$. perfringens, whereas a reduction of such domains was tolerated in non-spore-forming bacteria.

In mammalian SRP, the 9 (SRP9) and $14 \mathrm{kDa}$ (SRP14) subunits of SRP are required to confer elongation arrest activity to the particle. SRP9 and SRP14 form a heterodimer which specifically binds to Alu-like sequences, including domains I and II in 7SL RNA (Bovia et al., 1994; Strub \& Walter, 1990; Strub et al., 1991). In eubacteria, proteins other than Ffh that bind to scRNA have not been identified. According to the procedure for the purification of mammalian SRP (Walter \& Blobel, 1980), we are now purifying the RNP complex consisting of scRNA and the $\mathrm{Ffh}$ protein from $B$. subtilis. To characterize this complex should help to elucidate the functions of domains I and II.

\section{ACKNOWLEDGEMENTS}

We thank $\mathrm{H}$. Hayashi for providing Clostridium perfringens NCTC8237 and helpful discussion pertaining to this strain. We are grateful to N. Foster for critically reading the manuscript. This work was supported in part by grants-in-aid for scientific research from the Ministry of Education, Science and Culture of Japan.

\section{REFERENCES}

Althoff, S., Selinger, D. \& Wise, J. A. (1994). Molecular evolution of SRP cycle components: functional implications. Nucleic Acids Res 22, 1933-1947.

Altman, S. (1989). Ribonuclease P: an enzyme with catalytic RNA subunit. Adv Enzymol 62, 1-36.

Bernstein, H. D., Poritz, M. A., Strub, K., Hoben, P. J., Brenner, S. $\&$ Walter, P. (1989). Model for signal sequence recognition from amino acid sequence of $54 \mathrm{~K}$ subunit of signal recognition particle. Nature 340, 482-486.

Bikoff, E. \& Gefter, M. L. (1975). In vitro synthesis of transfer RNA. I. Purification of required components. J Biol Chem 250, 6240-6247.

Bourgaize, D. B. \& Fournier, M. J. (1987). Initiation of translation is impaired in E. coli cells deficient in 4.5S RNA. Nature 325, 281-284. 
Bovia, F., Bui, N. \& Strub, K. (1994). The heterodimeric subunit SRP9/14 of the signal recognition particle functions as permuted single polypeptide chain. Nucleic Acids Res 22, 2028-2035.

Brennwald, P., Liao, X., Holm, K., Porter, G. \& Wise, J. A. (1988). Identification of an essential Schizosaccharomyces pombe RNA homologous to the 7SL component of signal recognition particle. Mol Cell Biol 8, 1580-1590.

Brown, S. (1987). Mutations in the gene for EF-G reduce the requirement for 4.5S RNA in the growth of E. coli. Cell 49, 825-833.

Brown, S. (1989). Time of action of 4.5S RNA in E. coli translation. J Mol Biol 209, 79-90.

Brown, S. (1991). Genes for $7 \mathrm{~S}$ RNAs can replace the genes for $4.5 \mathrm{~S}$ RNA in growth of Escherichia coli. J Bacteriol 173, 1835-1837.

Brown, S. \& Fournier, M. J. (1984). The 4.5S RNA gene of E. coli is essential for cell growth. $J$ Mol Biol 178, 533-550.

Brown, S., Thon, G. \& Tolentino, E. (1989). Genetic selection and DNA sequences of 4.5S RNA homologs. J Bacteriol 171, 6517-6520.

Campos, N., Palau, J., Torrent, M. \& Ludevid, D. (1988). Signal recognition-like particles are present in maize. J Biol Chem 263, 9646-9650.

Deutscher, M. P. (1990). Ribonucleases, tRNA nucleotidyltransferase, and the $3^{\prime}$ processing of tRNA. Prog Nucleic Acid Res Mol Biol 39, 209-240.

Finegold, S. (1977). Anaerobic Bacteria in Human Disease. New York: Academic Press.

Fournier, M. J. \& Maxwell, E. (1993). The nucleolar snRNAs: catching up with the spliceosomal snRNAs. Trends Biochem Sci 18, 131-135.

Gilmore, R. (1993). Protein translocation across the endoplasmic reticulum: a tunnel with toll booths at entry and exit. Cell 75, 589-592.

Gitt, M. A., Wang, L. F. \& Doi, R. H. (1985). A strong sequence homology exists between RNA polymerase sigma factors of Bacillus subtilis and Eschericbia coli. J Biol Chem 260, 7178-7185.

Guthrie, C. (1991). Messenger RNA splicing in yeast: clues to why the spliceosome is a ribonucleoprotein. Science 253, 157-163.

Haas, E. S., Brown, J. W., Daniel, C. J. \& Reeve, J. N. (1990). Genes encoding the 7S RNA and tRNA ${ }^{\text {ser }}$ are linked to one of the two rRNA operons in the genome of the extremely thermophilic archaebacterium Methanothermus fervidus. Gene 90, 51-59.

Hann, B. C., Poritz, M. A. \& Walter, P. (1989). Saccharomyces cerevisiae and Schizosaccharomyces pombe contain a homologue to the $54 \mathrm{kD}$ subunit of the signal recognition particle that in $S$. cerevisiae is essential for growth. J Cell Biol 109, 3223-3230.

High, S. \& Dobberstein, B. (1991). The signal sequence interacts with the methionine-rich domain of the $54 \mathrm{kD}$ protein of signal recognition particle. J Cell Biol 113, 229-233.

Honda, K., Nakamura, K., Nishiguchi, M. \& Yamane, K. (1993). Cloning and characterization of a Bacillus subtilis gene encoding a homolog of the 54-kilodalton subunit of mammalian signal recognition particle and Escherichia coli Ffh. $J$ Bacteriol 175, 4885-4894.

Hoshino, T., Kose, K. \& Uratani, Y. (1990). Cloning and nucleotide sequence of the gene $b r a \mathrm{~B}$ coding for the sodium-coupled branchedchain amino acids carrier in Pseudomonas aeruginosa PAO. Mol \& Gen Genet 220, 461-467.

Inouye, M. \& Delihas, N. (1988). Small RNAs in the prokaryotes: a growing list of diverse roles. Cell 53, 5-7.

Kaine, B. P. (1990). Structure of the archaebacterial 7S RNA molecule. Mol \& Gen Genet 221, 315-321.
Krieg, U. C., Walter, P. \& Johnson, A. E. (1986). Photocrosslinking of the signal sequence of nascent preprolactin to the 54-kilodalton polypeptide of the signal recognition particle. Proc Natl Acad Sci US $A$ 83, 8604-8608.

Kurzchalia, T. V., Wiedmann, M., Girskovich, A. S., Bochkareva, E. S., Bielka, M. \& Rapoport, T. A. (1986). The signal sequence of nascent preprolactin interacts with the $54 \mathrm{~K}$ polypeptide of signal recognition particle. Nature 320,634-636.

Larsen, N. \& Zwieb, C. (1991). SRP-RNA sequence alignment and secondary structure. Nucleic Acids Res 19, 209-215.

van der Meer, J. R., Ludwig, W. \& des Vos, W. M. (1993). Characterization of a ribosomal RNA gene cluster from Clostridium tyroboturicum: phylogenetic positioning based on the 16S and 23S nucleotide sequences. Syst Appl Microbiol 16, 201-207.

Nakaar, V., Dare, A. O., Hong, D., Ullu, E. \& Tschudi, C. (1994). Upstream tRNA genes are essential for expression of small nuclear and cytoplasmic RNA genes in Trypanosomes. Mol Cell Biol 14, 6736-6742.

Nakamura, K., Nakamura, A., Takamatsu, H. \& Yamane, K. (1990). Cloning and characterization of a Bacillus subtilis gene homologous to Escherichia coli secY. J Biochem 107, 603-607.

Nakamura, K., Imai, Y., Nakamura, A. \& Yamane, K. (1992a). Small cytoplasmic RNA of Bacillus subtilis: functional relationship with human signal recognition particle 7S RNA and Escherichia coli $4.5 \mathrm{~S}$ RNA. J Bacteriol 174, 2185-2192.

Nakamura, K., Minemura, M., Nishiguchi, M., Honda, K. \& Yamane, K. (1992b). Conserved residues and secondary structure found in small cytoplasmic RNAs from Bacillus species. Nucleic Acids Res 20, 5227-5228.

Nakamura, K., Nishiguchi, M., Honda, K. \& Yamane, K. (1994). The Bacillus subtilis SRP54 homologue, $\mathrm{Ffh}$, has an intrinsic GTPase activity and forms a ribonucleoprotein complex with small cytoplasmic RNA in vivo. Biochem Biophys Res Commun 199, 1394-1399.

Nishiguchi, M., Honda, K., Amikura, R., Nakamura, K. \& Yamane, K. (1994). Structural requirements of Bacillus subtilis small cytoplasmic RNA for cell growth, sporulation, and extracellular enzyme production. J Bacteriol 176, 157-165.

Normanly, J., Ogden, R. C., Horvath, S. J. \& Abelson, J. (1986). Changing the identity of a transfer RNA. Nature 321, 213-219.

Nunnari, J. \& Walter, P. (1992). Protein targeting to and translocation across the membrane of the endoplasmic reticulum. Curr Opin Cell Biol 4, 573-580.

Poritz, M. A., Strub, K. \& Walter, P. (1988). Human SRP RNA and E. coli $4.5 S$ RNA contain a highly homologous structural domain. Cell 55, 4-6.

Rapoport, T. A. (1991). Protein transport across the endoplasmic reticulum membrane: facts, models, mysteries. FASEB J 5, 2792-2798.

Rapoport, T. A. (1992). Transport of proteins across the endoplasmic reticulum membrane. Science 258, 931-936.

Romish, K., Webb, J., Lingelbach, K., Gausepohl, H. \& Dobberstein, B. (1990). The $54-\mathrm{kD}$ protein of the signal recognition particle contains a methionine-rich RNA binding domain. $J$ Cell Biol 111, 1793-1802.

Saito, H. \& Miura, K. (1963). Preparation of transforming deoxyribonucleic acid by phenol treatment. Biochim Biopbys Acta 72, 619-629.

Samuelsson, T. (1992). Mycoplasma protein homologous to mammalian SRP54 recognizes a highly conserved domain of SRP RNA. Nucleic Acids Res 20, 5763-5770.

Samuelsson, T. \& Guindy, Y. (1990). Nucleotide sequence of a 
Mycoplasma mycoides RNA which is homologous to E. coli $4.5 \mathrm{~S}$ RNA. Nucleic Acids Res 18, 4938.

Samuelsson, T. \& Olsson, M. (1993). GTPase activity of a bacterial SRP-like complex. Nucleic Acids Res 21, 847-853.

Sanders, S. L. \& Schekman, R. (1992). Polypeptide translocation across the endoplasmic reticulum membrane. J Biol Chem 267, 13791-13794.

Sekiya, T., Contreras, R., Takeya, T. \& Khorana, H. G. (1979). Total synthesis of a tyrosine suppressor transfer RNA gene. XVII. Transcription in vitro of the synthetic gene and processing of the primary transcript to transfer RNA. J Biol Chem 254, 5802-5816.

Simoneau, P. \& Hu, P.-C. (1992). The 4.5S RNA homolog from Mycoplasma pneumoniae : genetic selection, sequence, and transcription analysis. $J$ Bacteriol 174, 627-629.

Smith, L. D. S. \& Hobbs, G. (1974). Clostridium. In Bergey's Manual of Determinative Bacteriology, pp. 551-572. Edited by R. E. Buchanan \& N. E. Gibbons. Baltimore: Williams \& Wilkins.

Strub, K. \& Walter, P. (1990). Assembly of the Alu domain of the signal recognition particle (SRP): dimerization of the two protein components is required for efficient binding to SRP RNA. Mol Cell Biol 10, 777-784.

Strub, K., Moss, J. C. B. \& Walter, P. (1991). Binding sites of the 9 and $14 \mathrm{kD}$ heterodimeric protein subunit of the signal recognition particle (SRP) are contained exclusively in the Alu domain of SRP RNA and contain a sequence motif that is conserved in evolution. Mol Cell Biol 11, 3949-3959.

Struck, J. C. R. \& Erdmannn, V. A. (1990). Phylogenetic and biochemical evidence for a secondary structure model of a small cytoplasmic RNA from Bacilli. Eur J Biochem 192, 17-24.

Struck, J. C. R., Toschka, H. Y. \& Erdmann, V. A. (1988a). Nucleotide sequence of the 4.5S RNA gene from Thermus thermopbilus HB8. Nucleic Acids Res 16, 9042.
Struck, J. C. R., Vogel, D. W., Ulbrich, N. \& Erdmann, V. A. (1988b). The Bacillus subtilis scRNA is related to the 4.5S RNA from Escherichia coli. Nucleic Acids Res 16, 2719.

Struck, J. C. R., Hartmann, R. K., Toschka, H. Y. \& Erdmann, V. A. (1989). Transcription and processing of Bacillus subtilis small cytoplasmic RNA. Mol \& Gen Genet 215, 478-482.

Tinoco, I., Borer, P. N., Dengler, B. \& Levine, M. D. (1973). Improved estimation of secondary structure in ribonucleic acids. Nat New Biol 246, 40-41.

Ullu, E. \& Weiner, A. M. (1984). Human genes and pseudogenes for the 7SL RNA component of signal recognition particle. EMBO J 3, 3303-3310.

Walter, P. \& Blobel, G. (1980). Purification of a membraneassociated protein complex required for protein translocation across the endoplasmic reticulum. Proc Natl Acad Sci US A 77, 7112-7116.

Walter, P. \& Blobel, G. (1982). 7SL RNA small cytoplasmic RNA is an integral component of the signal recognition particle. Nature 299, 691-698.

Walter, P. \& Lingappa, V. (1986). Mechanism of protein translocation across the endoplasmic reticulum membrane. Annu Rev Cell Biol 2, 499-516.

Woese, C. R. (1987). Bacterial evolution. Microbiol Rev 51, 221-271.

Zopf, D., Bernstein, H. D., Johnson, A. E. \& Walter, P. (1990). The methionine-rich domain of the $54 \mathrm{kd}$ protein subunit of the signal recognition particle contains an RNA binding site and can be crosslinked to a signal sequence. EMBO J 9, 4511-4517.

Zucker, M. (1989). Computer prediction of RNA structure. Methods Enzymol 180, 262-288.

Received 3 May 1995; revised 6 July 1995; accepted 13 July 1995. 